\title{
?19
}

TI 2019-064/II

Tinbergen Institute Discussion Paper

\section{The Shapley Value and Games with Hierarchies}

Encarnacion Algaba ${ }^{1}$

Rene van den Brink ${ }^{2}$

${ }^{1}$ Department of Applied Mathematics II and IMUS, Seville University, Spain

2 Department of Econometrics and Operations Research, and Tinbergen Institute, Vrije Universiteit Amsterdam, The Netherlands 
Tinbergen Institute is the graduate school and research institute in economics of Erasmus University Rotterdam, the University of Amsterdam and VU University Amsterdam.

Contact: discussionpapers@tinbergen.nl

More TI discussion papers can be downloaded at https://www.tinbergen.nl

Tinbergen Institute has two locations:

Tinbergen Institute Amsterdam

Gustav Mahlerplein 117

1082 MS Amsterdam

The Netherlands

Tel.: +31(0)205984580

Tinbergen Institute Rotterdam

Burg. Oudlaan 50

3062 PA Rotterdam

The Netherlands

Tel.: +31(0)10408 8900 


\title{
The Shapley Value and Games with Hierarchies
}

\author{
Encarnación Algaba ${ }^{1}$ René van den Brink ${ }^{2}$
}

September 5, 2019

${ }^{1}$ Department of Applied Mathematics II and IMUS, Seville University, Camino de los Descubrimientos s/n, 41092 Sevilla, Spain. E-mail: ealgaba@us.es.

${ }^{2}$ Department of Econometrics and Operations Research, and Tinbergen Institute, VU University Amsterdam, De Boelelaan 1105, 1081 HV Amsterdam, the Netherlands. E-mail: jrbrink@feweb.vu.nl. 


\begin{abstract}
In this paper we focus on restrictions arising from the players belonging to some hierarchical structure that is represented by a digraph. Two of these models are the games with a permission structure and games under precedence constraints. In both cases, the hierarchy can be represented by a directed graph which restricts the possibilities of coalition formation. These two approaches led to two different type of solutions in the literature. The precedence power solutions for games under precedence constraints, are axiomatized with an axiom that applies a network power measure to the precedence constraint. We will show that something similar can be done for games with a permission structure, and obtain a class of permission power solutions. This class contains the (conjunctive) permission value. With this we have two classes of solutions for games with a hierarchy, one based on permission structures and another based on precedence constraints, that are characterized by similar axioms. Moreover, the solutions are linked with network power measures.
\end{abstract}

Key words: Cooperative transferable utility game, permission structures, precedence constraints, Shapley value, hierarchical solution, power measures.

JEL code: C71 


\section{Introduction}

A situation in which a finite set of players can generate certain payoffs by cooperation can be described by a cooperative game with transferable utility (or simply a TU-game). A TU-game consists of a player set, and for every subset of the player set, called a coalition, a real number which is the worth that the coalition of players can earn when they agree to cooperate.

In a TU-game there are no restrictions on the cooperation possibilities of the players, i.e., every coalition is feasible and can generate a worth. Various models with restrictions on coalition formation are discussed in the literature. In this chapter, we focus on restrictions arising from the players belonging to some hierarchical structure that is represented by a digraph. Two of these models are the games with a permission structure introduced by Gilles et al. (1992) and games under precedence constraints introduced by Faigle and Kern (1992). In both cases, the hierarchy can be represented by a directed graph which restricts the possibilities of coalition formation. Whereas solutions for games with a permission structure are based on a restricted game that is defined from a set of feasible coalitions that typically is a proper subset of the power set of the full player set (i.e. it focuses on feasible combinations), in games under precedence constraints the coalition formation process is restricted in the sense that not all orders by which players enter a coalition can form (i.e. it focuses on feasible permutations). These two approaches led to two different type of solutions in the literature. In this chapter, we focus on acyclic digraphs.

In a game with a permission structure, the hierarchy or digraph is referred to as a permission structure, and this models the idea that there are players that need permission from other players before they are allowed to cooperate. Various assumptions can be made about how a permission structure affects the cooperation possibilities. In this chapter, we focus on the conjunctive approach, as developed in Gilles et al. (1992) and van den Brink and Gilles (1996), where it is assumed that every player needs permission from all its predecessors before it is allowed to cooperate. ${ }^{1}$

To take account of the limited cooperation possibilities, for every game with a permission structure a modified game is defined which assigns to every coalition the worth of its largest feasible subcoalition in the original game. A solution for games with a permission structure is a function that assigns to every such a game a payoff distribution over the individual players. Applying solutions for TU-games to the modified game yields solutions for games with a permission structure. In this chapter, we consider the Shapley

\footnotetext{
${ }^{1}$ Alternatively, for games with an acyclic quasi-strongly connected permission structure in the disjunctive approach, as considered in Gilles and Owen (1994) and van den Brink (1997), it is assumed that every player needs permission from at least one of its predecessors (if it has any) before it is allowed to cooperate with other players.
} 
value yielding a solution that is called the conjunctive (Shapley) permission value.

On the other hand, in a game under precedence constraints, the order in which players enter to form the 'grand coalition' is restricted by the digraph, in the sense, that players can only enter when their 'subordinates' in the hierarchy have already entered. Instead of taking the average of all marginal contribution vectors, as done by the classical Shapley value, the precedence (Shapley) value of Faigle and Kern (1992), takes the average of the marginal vectors over these admissible permutations. Alternatively, this precedence Shapley value can be written as an allocation of the Harsanyi dividends, where the dividend of every feasible coalition is allocated proportional to the so-called hierarchical strength being a power measure for digraphs that assigns to every player the number of admissible permutations where it is the last to enter. Algaba et al. (2017) showed that in this solution the payoff allocation is influenced by the presence of irrelevant players. These are players who do not generate worth in the game and, moreover, also all the players who depend on their presence do not generate worth in the game. Requiring that the payoff allocation does not depend on the presence of these irrelevant players, they modified the precedence Shapley value by requiring the allocation of Harsanyi dividends proportional to the hierarchical strength only in case all players are necessary to generate worth, (this means that the game is a multiple of the unanimity game of the 'grand coalition'). Moreover, they showed that instead of the hierarchical strength, any (positive) power measure can be used yielding the so-called precedence power solutions. In this way, the game theoretic problem of payoff allocation is linked with the social network literature on power and centrality measures.

After reviewing some known axiomatizations of the conjunctive permission value, the precedence Shapley value and precedence power solutions, we will show that also the conjunctive permission value can be axiomatized with an axiom that applies a network power measure to the permission structure. Moreover, similar as for the precedence power solutions, we can apply any (positive) power measure and obtain a class of permission power solutions. In this way, we have two classes of solutions for games with a hierarchy, one based on permission structures and another based on precedence constraints, that are characterized by similar axioms. Moreover, the solutions are linked with network power measures.

This chapter is organized as follows. After some preliminaries on cooperative transferable utility games and digraphs, in Section 2, we introduce the two models of games with a hierarchy. In Section 3, we discuss the conjunctive permission value, the precedence Shapley value and the hierarchical solution for these two models. In Section 4, we generalize these solutions by applying network power measures. In Section 5, we show logical independence of the axioms in the main theorems. Finally, Section 6 contains concluding remarks. 


\section{Games with Hierarchies}

\section{$2.1 \quad$ TU-games}

A situation in which a finite set of players $N \subset \mathbb{N}$ can generate certain payoffs by cooperation can be described by a cooperative game with transferable utility (or simply a TU-game), being a pair $(N, v)$ where $v: 2^{N} \rightarrow \mathbb{R}$ is a characteristic function on $N$ satisfying $v(\emptyset)=0$. For any coalition $S \subseteq N, v(S) \in \mathbb{R}$ is the worth of coalition $S$, i.e. the members of coalition $S$ can obtain a total payoff of $v(S)$ by agreeing to cooperate. We denote the collection of all TU-games $(N, v)$ by $\mathcal{G}$. We denote the collection of all characteristic functions $v$ on player set $N$ by $\mathcal{G}^{N}$.

A payoff vector for game $(N, v)$ is an $|N|$-dimensional vector $x \in \mathbb{R}^{N}$ assigning a payoff $x_{i} \in \mathbb{R}$ to any player $i \in N$. A (single-valued) solution for TU-games is a function that assigns a payoff vector to every TU-game. One of the most widely used solutions for TU-games is the Shapley value (Shapley (1953)), given by

$$
S h_{i}(N, v)=\frac{1}{|N| !} \sum_{\pi \in \Pi(N)} m_{i}^{\pi}(N, v) \text {, for all } i \in N
$$

where $\Pi(N)$ is the collection of all permutations $\pi: N \rightarrow N$ on $N$, and for every permutation $\pi \in \Pi(N)$,

$$
m_{i}^{\pi}(N, v)=v(\{j \in N \mid \pi(j) \leq \pi(i)\})-v(\{j \in N \mid \pi(j)<\pi(i)\}),
$$

is the marginal contribution of player $i$ to the players that are ranked before him in the order $\pi$.

For each $T \subseteq N, T \neq \emptyset$, the unanimity game $\left(N, u_{T}\right)$ is given by $u_{T}(S)=1$ if $T \subseteq S$, and $u_{T}(S)=0$ otherwise. It is well-known that the unanimity games form a basis for $\mathcal{G}^{N}$. For every $v \in \mathcal{G}^{N}$, it holds that $v=\sum_{\substack{T \subseteq N \\ T \neq \emptyset}} \Delta_{v}(T) u_{T}$, where $\Delta_{v}(T)=\sum_{S \subseteq T}(-1)^{|T|-|S|} v(S)$ are the Harsanyi dividends, see Harsanyi (1959).

For $(N, v),(N, w) \in \mathcal{G}$, the sum game $(N, v+w)$ is defined by $(v+w)(S)=v(S)+$ $w(S)$, and for $c \in \mathbb{R}$, the game $(N, c v) \in \mathcal{G}$ by $(c v)(S)=c v(S)$ for $S \subseteq N$. For $(N, v) \in \mathcal{G}$ and $S \subseteq N$, the subgame $\left(S, v_{S}\right)$ is given by $v_{S}(T)=v(T)$ for all $T \subseteq S$.

\subsection{Digraphs}

An irreflexive directed graph or irreflexive digraph is a pair $(N, D)$ where $N$ is the set of nodes and $D \subseteq\{(i, j) \mid i, j \in N, i \neq j\}$ is an (irreflexive) binary relation on $N$ consisting of ordered pairs called directed links or arcs. Since we assume irreflexivity throughout the full chapter, we refer to these just as digraphs. Since the nodes will represent players, we often 
refer to the nodes as players. For $i \in N$, the nodes in $F_{D}(i):=\{j \in N \mid(i, j) \in D\}$ are called the followers or successors of $i$ in $D$, and the nodes in $P_{D}(i):=\{j \in N \mid(j, i) \in D\}$ are called the predecessors of $i$ in $D$. Further, by $\widehat{F}_{D}(i)$ we denote the set of successors of $i$ in the transitive closure of $D$ i.e., $j \in \widehat{F}_{D}(i)$ if and only if there exists a sequence of players $\left(h_{1}, \ldots, h_{t}\right)$ such that $h_{1}=i, h_{k+1} \in F_{D}\left(h_{k}\right)$ for all $1 \leq k \leq t-1$, and $h_{t}=j$. We refer to the players in $\widehat{F}_{D}(i)$ as the subordinates of $i$ in $D$, and to the players in the set $\widehat{P}_{D}(i)=\left\{j \in N \mid i \in \widehat{F}_{D}(j)\right\}$ consisting of all predecessors of $i$ in the transitive closure of $D$, as $i$ 's superiors. We denote by $\widehat{P}_{D}(T)=\bigcup_{i \in T} \widehat{P}_{D}(i)$ the set of all superiors of players in $T$. The digraph $(N, D)$ is called acyclic if $i \notin \widehat{F}_{D}(i)$ for all $i \in N$. We denote the collection of all acyclic digraphs by $\mathcal{D}$, and the collection of all acyclic binary relations (which we will also often refer to as digraphs) on $N$ by $\mathcal{D}^{N}$. For $S \subseteq N$ and $(N, D) \in \mathcal{D}$, the digraph $(S, D(S))$ is given by $D(S)=\{(i, j) \in D \mid\{i, j\} \subseteq S\}$. By $T O P(N, D)=\left\{i \in N \mid P_{D}(i)=\emptyset\right\}$ we denote the set of 'top players' in $(N, D)$, i.e. the set of players without predecessors. Note that $\operatorname{TOP}(N, D) \neq \emptyset$ if $(N, D)$ is acyclic.

\subsection{Games with a Permission Structure}

A game with a permission structure describes a situation where some players in a TUgame need permission from other players before they are allowed to cooperate with other players. Formally, a permission structure is a directed graph on $N$. In this context, a triple $(N, v, D)$ with $N$ a finite set of players, $v \in \mathcal{G}^{N}$ a TU-game and $D \in \mathcal{D}^{N}$ a digraph on $N$ is called a game with a permission structure. In the conjunctive approach as introduced in Gilles et al. (1992) and van den Brink and Gilles (1996), it is assumed that a player needs permission from all its predecessors in order to cooperate with other players. In this sense, a coalition is feasible if and only if for every player in the coalition all its predecessors are also in the coalition. So, for permission structure $D$, the set of conjunctive feasible coalitions is given by

$$
\Phi^{c}(N, D)=\left\{S \subseteq N \mid P_{D}(i) \subseteq S \text { for all } i \in S\right\}
$$

For every $S \subseteq N$, let $\sigma_{D}^{c}(S)=\bigcup_{\left\{F \in \Phi^{c}(N, D) \mid F \subseteq S\right\}} F=S \backslash \widehat{F}_{D}(N \backslash S)$ be the largest conjunctive feasible subset ${ }^{2}$ of $S$ in the collection $\Phi^{c}(N, D)$. Then, the induced conjunctive restricted game of $(v, D)$ is the game $r_{v, D}^{c}: 2^{N} \rightarrow \mathbb{R}$ that assigns to every coalition $S \subseteq N$ the worth

\footnotetext{
${ }^{2}$ Every coalition having a unique conjunctive largest feasible subset follows from the fact that $\Phi^{c}(N, D)$ is union closed.
} 
of its largest conjunctive feasible subset ${ }^{3}$, i.e.

$$
r_{v, D}^{c}(S)=v\left(\sigma_{D}^{c}(S)\right) \text { for all } S \subseteq N
$$

We denote the class of all games with a permission structure by $\mathcal{G}_{P S}$.

\subsection{Games under Precedence Constraints}

Faigle and Kern (1992) consider situations where a partial order or acyclic directed graph represents a precedence relation meaning that the order in which players enter the grand coalition is restricted. Assuming that a player can only enter after all its subordinates have entered, a coalition is feasible if for every player in the coalition all of its successors in the digraph are also present in the coalition. The set $\Phi^{p}(N, D)$ of feasible coalitions according to digraph $(N, D) \in \mathcal{D}$ is thus given by

$$
\Phi^{p}(N, D)=\left\{S \subseteq N \mid F_{D}(i) \subseteq S \text { for all } i \in S\right\} .
$$

Instead of considering a restricted game on the collection of all coalitions (i.e. subsets of $N$ ), Faigle and Kern (1992) consider cooperative games, where for acyclic digraph $(N, D) \in \mathcal{D}$ the domain of the characteristic function is given by the set $\Phi^{p}(N, D)$. In this context, we call a triple $(N, v, D)$, where $N \subseteq \mathbb{N}$ is a finite set of players, $(N, D) \in \mathcal{D}$ is an acyclic digraph, and $v: \Phi^{p}(N, D) \rightarrow \mathbb{R}$, with $v(\emptyset)=0$, is a characteristic function that is defined only on $\Phi^{p}(N, D)$, a game under precedence constraints.

We denote the class of all games under precedence constraints by $\mathcal{G}_{P C}$, and we denote the class of games under precedence constraints on graph $(N, D) \in \mathcal{D}$ by $\mathcal{G}_{P C}^{(N, D)}$. For $(N, v, D),(N, w, D) \in \mathcal{G}_{P C}$, the sum game $(N, v+w)$ is defined by $(v+w)(S)=v(S)+w(S)$, and for $c \in \mathbb{R}$, the game $(N, c v) \in \mathcal{G}$ by $(c v)(S)=c v(S)$ for $S \in \Phi^{p}(N, D)$. The game under precedence constraints obtained from $(N, v, D) \in \mathcal{G}_{P C}$ by considering only feasible coalition $S$ and its subsets is denoted by $\left(S, v_{S}, D(S)\right)$, where $v_{S}(T)=v(T)$ for all feasible coalitions $T \subseteq S$. We refer to $\left(S, v_{S}, D(S)\right)$ as the subgame on $S$ of $(N, v, D)$.

Because of the difference in interpretation, we refer to a triple $(N, v, D)$ with $v$ a characteristic function on $2^{N}$ as a game with a permission structure, and to a triple $(N, v, D)$ with $v$ a characteristic function on $\Phi^{p}(N, D)$ as a game under precedence constraints. Sometimes, we refer to these situations in general as a game with a hierarchy.

\footnotetext{
${ }^{3}$ Alternatively, for acyclic and quasi-strongly connected permission structures, in the disjunctive approach as introduced in Gilles and Owen (1994) and van den Brink (1997) (see also Gilles (2010)), it is assumed that a non-top player needs permission from at least one of its predecessors. By a similar approach as described here, one can define the disjunctive restricted game.
} 


\section{Solutions for Games with Hierarchies}

\subsection{The Conjunctive Permission Value for Games with a Per- mission Structure}

A solution for games with a permission structure is a function $f$ that assigns a payoff distribution $f(N, v, D) \in \mathbb{R}^{N}$ to every game with permission structure $(N, v, D)$. The conjunctive (Shapley) permission value $\varphi^{c}$ is the solution that assigns to every game with a permission structure the Shapley value of the conjunctive restricted game ${ }^{4}$, i.e.

$$
\varphi^{c}(N, v, D)=\operatorname{Sh}\left(N, r_{v, D}^{c}\right)
$$

Next, we discuss one of the axiomatizations of the conjunctive permission value. Player $i \in N$ is inessential in game with permission structure $(N, v, D)$ if $i$ and all its subordinates are null players in game $v$, i.e., if $v(S)=v(S \backslash\{j\})$ for all $S \subseteq N$ and $j \in\{i\} \cup \widehat{F}_{D}(i)$. Player $i \in N$ is necessary in game $v$ if $v(S)=0$ for all $S \subseteq N \backslash\{i\}$.

Next, we mention some axioms of solutions for games with a permission structure. Efficiency and linearity are straightforward generalizations of TU-game solution axioms. The inessential player property requires that inessential players earn a zero payoff. The necessary player property requires that necessary players earn at least as much as any other player if the game is monotone. Notice that a necessary player is a 'strong' player in a monotone game. Structural monotonicity requires that in monotone games, players earn at least as much as their successors. From now on, the class of monotone TU-games on $N$ will be denoted by $\mathcal{G}_{M}^{N}$.

Efficiency For every $v \in \mathcal{G}^{N}$ and $D \in \mathcal{D}^{N}$, it holds that $\sum_{i \in N} f_{i}(N, v, D)=v(N)$.

Linearity For every $v, w \in \mathcal{G}^{N}$ and $D \in \mathcal{D}^{N}$, it holds that $f(N, v+w, D)=f(N, v, D)+$ $f(N, w, D)$, and for $c \in \mathbb{R}$ it holds that $f(N, c v, D)=c f(N, v, D)$.

Inessential player property For every $v \in \mathcal{G}^{N}$ and $D \in \mathcal{D}^{N}$, if $i \in N$ is an inessential player in $(N, v, D)$ then $f_{i}(N, v, D)=0$.

Necessary player property For every $v \in \mathcal{G}_{M}^{N}$ and $D \in \mathcal{D}^{N}$, if $i \in N$ is a necessary player in $(N, v)$ then $f_{i}(N, v, D) \geq f_{j}(N, v, D)$ for all $j \in N$.

Structural monotonicity For every $v \in \mathcal{G}_{M}^{N}$ and $D \in \mathcal{D}^{N}$, if $j \in F_{D}(i)$ then $f_{i}(N, v, D) \geq$ $f_{j}(N, v, D)$.

\footnotetext{
${ }^{4}$ Alternatively, for acyclic and quasi-strongly connected permission structures, the disjunctive permission value is obtained as the Shapley value of the disjunctive restricted game, see Footnote 3.
} 
The above five axioms characterize the conjunctive permission value. ${ }^{5}$

Theorem 3.1 (van den Brink and Gilles (1996)) A solution for games with a permission structure is equal to the conjunctive permission value $\varphi^{c}$ if and only if it satisfies efficiency, linearity, the inessential player property, the necessary player property and structural monotonicity.

If $D=\emptyset$ then there are no restrictions in coalition formation (i.e. $\Phi^{c}(N, D)=2^{N}$ ), and then $\varphi^{c}(N, v, D)=S h(N, v)$. In this sense, the conjunctive permission value generalizes the Shapley value for TU-games. Notice that the axiomatization in Theorem 3.1 gives an axiomatization of the Shapley value for TU-games by taking $D=\emptyset$. In that case, efficiency and linearity just boil down to the corresponding axioms for TU-game solutions. Since no player has subordinates, a player is inessential if and only if it is a null player in the game, and thus the inessential player property boils down to the null player property for TU-game solutions. The necessary player property does not depend on the permission structure anyway, and can be stated as well for TU-game solutions by requiring that a necessary player in a monotone game earns at least as much as any other player. ${ }^{6}$ Efficiency, linearity, the inessential (null) player property and the necessary player property then give uniqueness as in Shapley (1953). Note that structural monotonicity has no meaning when $D=\emptyset$

In Gilles at al. (1992) it is shown that the conjunctive permission value can also be obtained by allocating the Harsanyi dividends in the conjunctive restricted game, equally over all players in the corresponding coalition and their superiors, i.e.

$$
\varphi_{i}^{c}(N, v, D)=\sum_{\substack{S \subseteq N \\ i \in S \cup \widehat{P}_{D}(S)}} \frac{\Delta_{r_{v, D}^{c}}(S)}{\left|S \cup \widehat{P}_{D}(S)\right|} \text { for all } i \in N
$$

\subsection{The Precedence Shapley Value and the Hierarchical Solution for Games under Precedence Constraints}

\subsubsection{The Precedence Shapley Value}

Faigle and Kern (1992) introduce the precedence Shapley value as solution for games under precedence constraints. First, a permutation $\pi \in \Pi(N)$ is called admissible in acyclic digraph $(N, D)$ if $\pi(i)>\pi(j)$ whenever $(i, j) \in D$, i.e. successors enter before their

\footnotetext{
${ }^{5}$ We remark that, instead of linearity van den Brink and Gilles (1996) use the weaker additivity axiom.

${ }^{6}$ Since all players in $T \subseteq N$ are necessary players in the unanimity game $u_{T}$ on $T$, they should earn the same in that unanimity game, which in the axiomatization of the Shapley value is guaranteed by symmetry.
} 
predecessors in the digraph. ${ }^{7}$ The set of admissible permutations $\Pi_{D}(N)$ in $D$ is denoted by

$$
\Pi_{D}(N)=\{\pi \in \Pi(N) \mid \pi(i)>\pi(j) \text { if }(i, j) \in D\}
$$

Note that the set of admissible permutations in $D$ is the same as that of its transitive closure $\operatorname{tr}(D): \Pi_{D}(N)=\Pi_{\operatorname{tr}(D)}(N)$.

The precedence marginal vector $m^{\pi}(N, v, D) \in \mathbb{R}^{N}$, associated with the game under precedence constraints $(N, v, D)$, permutation $\pi \in \Pi_{D}(N)$, and player $i \in N$, is given by

$$
m_{i}^{\pi}(N, v, D)=v(\{j \in N \mid \pi(j) \leq \pi(i)\})-v(\{j \in N \mid \pi(j)<\pi(i)\}) .
$$

Recall from Section 2 that the Shapley value assigns to the players the average over all marginal vectors associated with all permutations of the player set $N$. The precedence Shapley value $H$ is the solution on $\mathcal{G}_{P C}$ given by

$$
H_{i}(N, v, D)=\frac{1}{\left|\Pi_{D}(N)\right|} \sum_{\pi \in \Pi_{D}(N)} m_{i}^{\pi}(N, v, D), \quad \text { for all } i \in N
$$

and assigns to the players in $N$ the average over all precedence marginal vectors of game under precedence constraints $(N, v, D)$. For $(N, v, D) \in \mathcal{G}_{P C}$, all permutations in $\Pi(N)$ are admissible when $D=\emptyset$. In that case the domain of characteristic function $v$ is given by $2^{N}$, and thus is a classical characteristic function of a TU-game. So, also the precedence Shapley value $H$ generalizes the Shapley value for TU-games.

Faigle and Kern (1992) give an axiomatization of the precedence Shapley value using the following axioms. Efficiency and linearity are the same as for the conjunctive permission value, but defined on the domain $\mathcal{G}_{P C}$.

Efficiency For each game $(N, v, D) \in \mathcal{G}_{P C}$ it holds that $\sum_{i \in N} f_{i}(N, v, D)=v(N)$.

Linearity For every pair of games $(N, v, D)$ and $(N, w, D) \in \mathcal{G}_{P C}^{(N, D)}$ it holds that $f(N, v+$ $w, D)=f(N, v, D)+f(N, w, D)$, and for $(N, v, D) \in \mathcal{G}_{P C}^{(N, D)}$ and $c \in \mathbb{R}$ it holds that $f(N, c v, D)=c f(N, v, D)$.

A player $i \in N$ is a null player in game under precedence constraints $(N, v, D)$, if for every $\pi \in \Pi_{D}(N)$ it holds that $m_{i}^{\pi}(N, v, D)=0$.

Null player property For each $(N, v, D) \in \mathcal{G}_{P C}$, if $i \in N$ is a null player in $(N, v, D)$, then $f_{i}(N, v, D)=0$.

\footnotetext{
${ }^{7}$ The terminology looks somewhat counterintuitive, but this is because of the different interpretations of the hierarchy in games with a permission structure and games under precedence constraints, see also the last paragraph of Section 6.
} 
Besides these three axioms ${ }^{8}$, Faigle and Kern (1992) introduce an axiom that is based on the hierarchical strength of players. First, for all $i \in S, S \in \Phi^{p}(N, D)$, the set of permutations $\Pi_{D}^{i}(N, S)$ is defined by

$$
\Pi_{D}^{i}(N, S)=\left\{\pi \in \Pi_{D}(N) \mid \pi(i)>\pi(j) \text { for all } j \in S \backslash\{i\}\right\}
$$

being the collection of those admissible permutations in $\Pi_{D}(N)$ where $i$ enters after the players in $S \backslash\{i\}$. Note that the collection $\left\{\Pi_{D}^{i}(N, S)\right\}_{i \in S}$ is a partition of $\Pi_{D}(N)$.

The absolute hierarchical strength is the function $h$ that assigns to every $(N, D) \in \mathcal{D}$ and coalition $S \in \Phi^{p}(N, D)$, the vector $h(N, D, S) \in \mathbb{R}^{S}$, where $h_{i}(N, D, S)=\left|\Pi_{D}^{i}(N, S)\right|$ is the number of permutations in $\Pi_{D}(N)$ where $i \in S$ enters after the players in $S \backslash\{i\}$.

The normalized hierarchical strength is the function $\bar{h}$ that assigns to every $(N, D) \in$ $\mathcal{D}$ and coalition $S \in \Phi^{p}(N, D)$, the vector $\bar{h}(N, D, S) \in \mathbb{R}^{S}$, where $\bar{h}_{i}(N, D, S)=\frac{\left|\Pi_{D}^{i}(N, S)\right|}{\left|\Pi_{D}(N)\right|}$ is the fraction of permutations in $\Pi_{D}(N)$ where $i \in S$ enters after the players in $S \backslash\{i\}$. Note that $\sum_{i \in S} \bar{h}_{i}(N, D, S)=1$ for all $S \in \Phi^{p}(N, D)$.

Unanimity games under precedence constraints are defined similar to classical unanimity TU-games. For each $T \in \Phi^{p}(N, D), T \neq \emptyset$, the unanimity game under precedence constraints $\left(N, u_{T}, D\right) \in \mathcal{G}_{P C}$ is given by $u_{T}(S)=1$ if $T \subseteq S$, and $u_{T}(S)=0$ otherwise, $S \in \Phi^{p}(N, D)$. Note that, different from classical TU-games, the unanimity game (called simple game by Faigle and Kern) $u_{T}$ is only defined on the set $\Phi^{p}(N, D)$. Faigle and Kern (1992) also consider the dividend of a coalition $S \in \Phi^{p}(N, D)$ in game under precedence constraints $(N, v, D)$, given by $\Delta_{v}^{D}(S)=v(S)-\sum_{T \subset S, T \in \Phi^{p}(N, D), T \neq \emptyset} \Delta_{v}^{D}(T)$.

For every $(N, v, D) \in \mathcal{G}_{P C}$, Faigle and Kern (1992) show that the characteristic function in $(N, v, D)$ can be written as a linear combination of the characteristic functions of unanimity games under precedence constraints $\left(N, u_{T}, D\right)$ :

$$
v=\sum_{\substack{T \in \Phi^{p}(N, D) \\ T \neq \emptyset}} \Delta_{v}^{D}(T) u_{T} .
$$

The axiom of hierarchical strength of a solution for games under precedence constraints states that in unanimity games under precedence constraints, the earnings are distributed among the players in the unanimity coalition proportional to their normalized hierarchical strength in that coalition. Obviously, this is equivalent to distributing the dividends proportional to the absolute hierarchical strength of the players.

Hierarchical strength For every $(N, D) \in \mathcal{D}$, every $S \in \Phi^{p}(N, D)$ and every $i, j \in S$, it holds that $\bar{h}_{i}(N, D, S) f_{j}\left(N, u_{S}, D\right)=\bar{h}_{j}(N, D, S) f_{i}\left(N, u_{S}, D\right)$.

\footnotetext{
${ }^{8}$ We remark that, similar to Shapley (1953), Faigle and Kern (1992) combine efficiency and the null player property into a carrier axiom.
} 
Theorem 3.2 (Faigle and Kern, 1992) A solution on $\mathcal{G}_{P C}$ is equal to the precedence Shapley value $H$ if and only if it satisfies efficiency, linearity, the null player property and hierarchical strength.

Alternatively, the precedence Shapley value can be defined as the solution that allocates the dividend of a coalition $S \in \Phi^{p}(N, D)$ proportional to the hierarchical strength $h(N, D, S)$ of the players in $S$ :

$$
H_{i}(N, v, D)=\sum_{\substack{S \in \Phi,(N, D) \\ i \in S}} \frac{h_{i}(N, D, S)}{\sum_{j \in S} h_{j}(N, D, S)} \Delta_{v}^{D}(S) \text { for all } i \in N
$$

\subsubsection{The Hierarchical Solution}

Algaba et al. (2017) introduce the solution for games under precedence constraints that is obtained by weakening hierarchical strength in Theorem 3.2 to weak hierarchical strength and adding irrelevant player independence.

Weak hierarchical strength is a weaker version of the hierarchical strength axiom in the sense that it only requires the equality for unanimity games of the grand coalition.

Weak hierarchical strength For every $(N, D) \in \mathcal{D}$ and every $i, j \in N$, it holds that $\bar{h}_{i}(N, D, N) f_{j}\left(N, u_{N}, D\right)=\bar{h}_{j}(N, D, N) f_{i}\left(N, u_{N}, D\right)$.

This is a considerable weakening, also in interpretation. If unanimity among all players must be reached before any non-zero worth can be generated, we might consider the players equals with respect to the game. Therefore, worth allocation should depend only on the strength of the players in the digraph. The strength of each player in the digraph is measured by the hierarchical strength.

Player $i \in N$ is called an irrelevant player in game under precedence constraints $(N, v, D)$ if $i$ is a null player, and any $j \in \widehat{P}_{D}(i)$ is also a null player (this implies that any $j \in \widehat{P}_{D}(i)$ is also irrelevant). So, an irrelevant player is a null player such that all players who depend on its presence are also null players in the game. We call a player $i \in N$ relevant if it is not an irrelevant player.

Let $\operatorname{Irr}(N, v, D)$ be the set of irrelevant players in game under precedence constraints $(N, v, D)$. Irrelevant player independence states that removal of irrelevant players from the game, does not affect the payoff to relevant players.

Irrelevant player independence For every $(N, v, D) \in \mathcal{G}_{P C}$, it holds that $f_{i}(N, v, D)=$ $f_{i}\left(N^{\prime}, v_{N^{\prime}}, D\left(N^{\prime}\right)\right)$ for $i \in N^{\prime}$, with $N^{\prime}=N \backslash \operatorname{Irr}(N, v, D)$. 
For a collection of sets $\mathcal{F} \subseteq 2^{N}$, let $\mathcal{F}_{S}=\{T \in \mathcal{F} \mid T \subseteq S\}$ be the collection of subsets of $S$ in $\mathcal{F}$. It can be seen that, for $N^{\prime}=N \backslash \operatorname{Irr}(N, v, D)$, it holds that $\Phi_{N^{\prime}}^{p}(N, D)=$ $\Phi^{p}\left(N^{\prime}, D\left(N^{\prime}\right)\right)$, i.e. the collection of feasible subsets of coalition $N^{\prime}$ obtained from graph $(N, D)$ is equal to the collection of feasible sets obtained from subgraph $\left(N^{\prime}, D\left(N^{\prime}\right)\right.$ ). (Note that this does not have to be the case for all subsets of $N)$. This means that removing irrelevant players from the game does not have an effect on the ability of relevant players to cooperate with each other.

We consider irrelevant player independence a desirable property for a solution for games under precedence constraints to satisfy. Since irrelevant players are null players, they do not make any contribution to their subordinates in the digraph. Moreover, their superiors are also null players, and thus irrelevant players do not make any contribution through players that need them to be present in any admissible permutation. Therefore, they should not be able to affect the payoffs of those players who do make a contribution in the game. The precedence Shapley value does not satisfy irrelevant player independence, as illustrated by the following example.

Example 3.3 Consider the following acyclic digraph used by Faigle and Kern (1992). Let $N=\{1,2,3,4\}$, and $D=\{(3,1),(3,2),(4,2)\}$.

The set of admissible permutations is

$$
\Pi_{D}(N)=\{(1,2,3,4),(1,2,4,3),(2,1,3,4),(2,1,4,3),(2,4,1,3)\} .
$$

In this case, for $S=\{1,2,4\} \in \Phi^{p}(N, D)$, we have

$$
h_{1}(N, D, S)=1, h_{2}(N, D, S)=0, h_{4}(N, D, S)=4
$$

Consider the game $v=u_{\{1,2,4\}}$. Then, the precedence Shapley value is given by $H(N, v, D)=$ $\left(\frac{1}{5}, 0,0, \frac{4}{5}\right)$.

Notice that player 3 is an irrelevant player. Deleting player 3 gives the game under precedence constraints $\left(N^{\prime}, v^{\prime}, D^{\prime}\right)$ with $N^{\prime}=\{1,2,4\}, v^{\prime}=u_{\{1,2,4\}}$ (but on a different domain), and $D^{\prime}=\{(4,2)\}$.

The set of admissible permutations on subgraph $(S, D(S))$ is given by

$$
\Pi_{D(S)}(S)=\{(1,2,4),(2,1,4),(2,4,1)\} .
$$

Therefore, $h_{1}(S, D(S), S)=1, h_{2}(S, D(S), S)=0, h_{4}(S, D(S), S)=2$, yielding the precedence Shapley value $H(N, v, D)=\left(\frac{1}{3}, 0, \frac{2}{3}\right)$.

The presence of irrelevant player 3 changes the payoffs of players 1 and 4 according to the precedence Shapley value. 


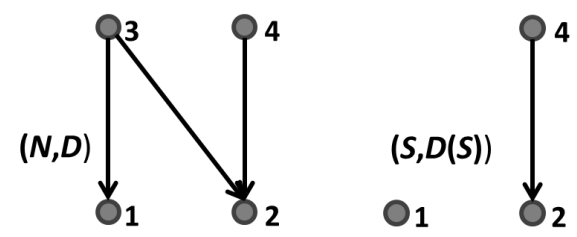

Figure 1: Digraphs $(N, D)$ and $(S, D(S))$ of Example 3.3

It can be shown that for games $\left(N_{m}, u_{\{1,2\}}, D_{m}\right)$, where $N_{m}$ is given by $\{1, \ldots, m\}$ and $D_{m}$ by $\{(3,1),(4,3), \ldots,(m, m-1)\}$, the precedence Shapley value is given by $H_{1}\left(N_{m}, u_{\{1,2\}}, D_{m}\right)=$ $\frac{1}{m}, H_{2}\left(N_{m}, u_{\{1,2\}}, D_{m}\right)=\frac{m-1}{m}$ and $H_{i}\left(N_{m}, u_{\{1,2\}}, D_{m}\right)=0$ for $i \in N_{m} \backslash\{1,2\}$ and so $\lim _{m \rightarrow \infty} H_{1}\left(N_{m}, u_{\{1,2\}}, D_{m}\right)=0$ and $\lim _{m \rightarrow \infty} H_{2}\left(N_{m}, u_{\{1,2\}}, D_{m}\right)=1$. We find that the fact that player 1 has many irrelevant players as superiors in the digraph, is detrimental to its payoff, even though, for different values of $m$, player 1 is present in exactly the same feasible coalitions that contain only relevant players.

Algaba et al. (2017) provide a characterization where the null player property is replaced by the following weaker property on irrelevant players. ${ }^{9}$

Irrelevant player property For each $(N, v, D) \in \mathcal{G}_{P C}$, if $i \in N$ is an irrelevant player in $(N, v, D)$, then $f_{i}(N, v, D)=0$.

The unique solution for games under precedence constraints that satisfies efficiency, linearity, the irrelevant player property, irrelevant player independence, and weak hierarchical strength is the hierarchical solution which allocates the dividend of every feasible coalition over the players in that coalition proportional to the hierarchical strength in the subgraph on that coalition.

The hierarchical solution $\widetilde{H}$ is the solution on $\mathcal{G}_{P C}$ given by

$$
\widetilde{H}_{i}(N, v, D)=\sum_{\substack{S \in \Phi^{p}(N, D) \\ i \in S}} \frac{h_{i}(S, D(S), S)}{\sum_{j \in S} h_{j}(S, D(S), S)} \Delta_{v}^{D}(S), i \in N .
$$

A main difference with the precedence Shapley value is that in that value, the dividends are allocated proportional to the hierarchical strength in the full digraph (see (3.7)), while in the hierarchical solution, when allocating the dividend of a feasible coalition, we consider the hierarchical strength of the subgraph on the corresponding coalition.

Theorem 3.4 (Algaba et al., 2017) A solution for games under precedence constraints is equal to the hierarchical solution $\widetilde{H}$ if and only if it satisfies efficiency, linearity, the irrelevant player property, irrelevant player independence, and weak hierarchical strength.

\footnotetext{
${ }^{9}$ It is straightforward to show that the null player property can also be replaced by the irrelevant player property in the axiomatization of the precedence Shapley value.
} 
Next, we provide an example which calculates the hierarchical solution and the precedence Shapley value highlighting that in general both solutions are different, and are also different from the conjunctive permission value.

Example 3.5 Consider the game under precedence constraint given in Example 3.3. By taking the appropriate domain for the characteristic function, this can also be seen as a game with a permission structure. In Example 3.3, we already computed the precedence Shapley value $H(N, v, D)=\left(\frac{1}{5}, 0,0, \frac{4}{5}\right)$.

For $S=\{1,2,4\} \in \Phi^{p}(N, D)$, in Example 3.3, we also found that the set of admissible permutations on subgraph $(S, D(S))$ is given by

$$
\Pi_{D(S)}(S)=\{(1,2,4),(2,1,4),(2,4,1)\}
$$

and thus $h_{1}(S, D(S), S)=1, h_{2}(S, D(S), S)=0, h_{4}(S, D(S), S)=2$. This yields the hierarchical solution $\widetilde{H}(N, v, D)=\left(\frac{1}{3}, 0,0, \frac{2}{3}\right)$.

The conjunctive restricted game is given by $r_{v, D}^{c}=u_{N}$, and thus the conjunctive permission value gives an equal allocation of the payoff, $\varphi^{c}(N, v, D)=\left(\frac{1}{4}, \frac{1}{4}, \frac{1}{4}, \frac{1}{4}\right)$.

\section{Power Measures for Digraphs and Solutions for Games with Hierarchies}

Power or centrality measures for digraphs are applied to define solutions for games under precedence constraints by Algaba et al. (2017). In this section, we first review their result, and then apply this approach to games with a permission structure.

\subsection{Precedence Power Solutions for Games under Precedence Constraints}

Algaba et al. (2017) considered a class of solutions for games under precedence constraints that contains the hierarchical solution. Similar as van den Brink et al. (2011a) generalize the communication ability property of Borm et al. (1992) for communication graph games (see Myerson (1977)), this class is obtained by applying a power measure for digraphs to allocate the dividends, and apply this power measure in a corresponding version of the weak hierarchical strength axiom.

A power measure for acyclic digraphs is a function $p$, that to every acyclic digraph $(N, D) \in \mathcal{D}$ assigns a vector $p(N, D) \in \mathbb{R}^{N}$. For a player $i \in N, p_{i}(N, D)$ is a measure of its relational 'power' or 'influence' in $(N, D)$. We call a power measure $p$ positive if $\sum_{j \in N} p_{j}(N, D)>0$ for all $(N, D) \in \mathcal{D}$ with $D \neq \emptyset$. Notice that a power measure is defined 
for any set of nodes $N \subset \mathbb{N}$, and thus also for any $S \subseteq N \subset \mathbb{N}, p(S, D(S))$ is defined. In this chapter, we only consider positive power measures. Let the collection of all positive power measures be denoted by $P$.

For positive power measure $p$, we define the $p$-hierarchical solution as the solution that allocates the dividend of a coalition $S \in \Phi^{p}(N, D)$ among the players in $S$ proportional to $p(S, D(S))$.

Definition 4.1 For positive power measure $p$, the p-hierarchical solution is the solution on $\mathcal{G}_{P C}$ given by

$$
H_{i}^{p}(N, v, D)=\sum_{\substack{S \in \Phi^{p}(N, D) \\ i \in S}} \frac{p_{i}(S, D(S))}{\sum_{j \in S} p_{j}(S, D(S))} \Delta_{v}^{D}(S) \text { for all } i \in N .
$$

We refer to the class consisting of all $p$-hierarchical solutions as the class of precedence power solutions.

In order to axiomatize the $p$-hierarchical solution, the $p$-strength axiom is introduced. This axiom has an interpretation similar to that of weak hierarchical strength from Theorem 3.4. If unanimity among all players must be reached to generate any nonzero worth, we might consider the players equals with respect to the game. Therefore, worth allocation should only depend on the strength of the players in the digraph. The $p$-hierarchical solution uses the power measure $p$ to measure the strength of each player in the digraph. The $p$-strength axiom requires that in a game where all players are necessary to generate worth, the payoffs are allocated proportional to the power measure $p$.

$p$-strength Let $p$ be a positive power measure. For every $(N, D) \in \mathcal{D}$ and every $i, j \in N$, it holds that

$p_{i}(N, D) f_{j}\left(N, u_{N}, D\right)=p_{j}(N, D) f_{i}\left(N, u_{N}, D\right)$.

The $p$-hierarchical solution is axiomatized by replacing in Theorem 3.4 weak hierarchical strength by $p$-strength.

Theorem 4.2 (Algaba et al., 2017) A solution for games under precedence constraints is equal to the p-hierarchical solution $H^{p}$ if and only if it satisfies efficiency, linearity, the irrelevant player property, irrelevant player independence and p-strength.

Note that this gives Theorem 3.4 as a corollary by taking the hierarchical strength as power measure. The axioms of Theorem 4.2 are not logically independent. It can be shown that efficiency and irrelevant player independence together imply the irrelevant player property. 
Proposition 4.3 Consider a solution $f$ on $\mathcal{G}_{P C}$. If $f$ satisfies efficiency and irrelevant player independence, then $f$ satisfies the irrelevant player property.

Proof: Suppose that solution $f$ satisfies efficiency and irrelevant player independence. We show that $f$ must satisfy the irrelevant player property by induction on the number of irrelevant players. Suppose that $|\operatorname{Irr}(N, v, D)|=1$, and let $j \in N$ be the irrelevant player in $(N, v, D) \in \mathcal{G}_{P C}$. By irrelevant player independence, we have that $f_{i}(N, v, D)=$ $f_{i}\left(N \backslash\{j\}, v_{N \backslash\{j\}}, D(N \backslash\{j\})\right)$ for all $i \in N \backslash\{j\}$. By efficiency it then follows that $\sum_{i \in N} f_{i}(N, v, D)=v(N)=v(N \backslash\{j\})=\sum_{i \in N \backslash\{j\}} f_{i}\left(N \backslash\{j\}, v_{N \backslash\{j\}}, D(N \backslash\{j\})\right)=$ $\sum_{i \in N \backslash\{j\}} f_{i}(N, v, D)$, and thus $f_{j}(N, v, D)=0$.

By induction, we assume that irrelevant players get a zero payoff for all $\left(N^{\prime}, v^{\prime}, D^{\prime}\right) \in \mathcal{G}_{P C}$ with $\left|\operatorname{Irr}\left(N^{\prime}, v^{\prime}, D^{\prime}\right)\right|<|\operatorname{Irr}(N, v, D)|$. Take a $j \in \operatorname{Irr}(N, v, D)$ such that all successors of $j$ are relevant players. (Existence of such players can be shown as follows. Consider an irrelevant player who has an irrelevant successor. If this successor has at least one irrelevant successor, then consider this successor. Continuing in this way, by acyclicity and finiteness of $D$, eventually we reach an irrelevant player whose successors are all relevant players, possibly being an irrelevant player who has no successors.) Consider the game $w=$ $u_{(N \backslash \operatorname{Irr}(N, v, D)) \cup\{j\}}$, i.e. the unanimity game on the set of all relevant players in $v$ with player $j$. Then $|\operatorname{Irr}(N, w, D)|<|\operatorname{Irr}(N, v, D)|$, specifically $\operatorname{Irr}(N, w, D)=\operatorname{Irr}(N, v, D) \backslash\{j\}$, and thus by the induction hypothesis

$$
\sum_{h \in \operatorname{Irr}(N, v, D) \backslash\{j\}} f_{h}(N, w, D)=0 .
$$

Since also $|\operatorname{Irr}(N, v+w, D)|=|\operatorname{Irr}(N, w, D)|=|\operatorname{Irr}(N, v, D)|-1$, we have similar that

$$
\sum_{h \in \operatorname{Irr}(N, v, D) \backslash\{j\}} f_{h}(N, v+w, D)=0 .
$$

Since linearity implies that $f(N, v, D)=f(N, v+w, D)-f(N, w, D)$, with (4.8) and (4.9), it follows that

$$
\begin{aligned}
\sum_{h \in \operatorname{Irr}(N, v, D) \backslash\{j\}} f_{h}(N, v, D) & =\sum_{h \in \operatorname{Irr}(N, v, D) \backslash\{j\}} f_{h}(N, v+w, D) \\
& -\sum_{h \in \operatorname{Irr}(N, v, D) \backslash\{j\}} f_{h}(N, w, D)=0 .
\end{aligned}
$$

By efficiency and irrelevant player independence it follows, similar as in the initial step, that $\sum_{i \in N} f_{i}(N, v, D)=v(N)=v(N \backslash \operatorname{Irr}(N, v, D))=$ 
$=\sum_{i \in N \backslash \operatorname{Irr}(N, v, D)} f_{i}\left(N \backslash \operatorname{Irr}(N, v, D), v_{N \backslash \operatorname{Irr}(N, v, D)}, D(N \backslash \operatorname{Irr}(N, v, D))\right)=\sum_{i \in N \backslash \operatorname{Irr}(N, v, D)} f_{i}(N, v, D)$, and thus $\sum_{h \in \operatorname{Irr}(N, v, D)} f_{h}(N, v, D)=0$. With (4.10) it then follows that $f_{j}(N, v, D)=0$. This shows that the irrelevant player property is satisfied.

Proposition 4.3 and Theorem 4.2 immediately give the following result as a corollary. ${ }^{10}$

Theorem 4.4 A solution for games under precedence constraints is equal to the p-hierarchical solution $H^{p}$ if and only if it satisfies efficiency, linearity, irrelevant player independence and p-strength.

Logical independence of the axioms is shown in Section 5.

\subsection{Power Measures, Solutions for Games with a Permission Structure and Permission Values}

The conjunctive permission value satisfies efficiency and linearity ${ }^{11}$. Although the conjunctive permission value does not satisfy the weak hierarchical strength axiom, it satisfies a version of the $p$-strength axiom, where in the unanimity game of the 'grand coalition', the payoffs are allocated equally over the players, i.e. proportional to the equal power measure where all players have equal power in any digraph. ${ }^{12}$

Equal-strength For every $(N, D) \in \mathcal{D}$ and every $i, j \in N$, it holds that $f_{i}\left(N, u_{N}, D\right)=$ $f_{j}\left(N, u_{N}, D\right)$.

The conjunctive permission value does not satisfy the null player property, irrelevant player independence and the irrelevant player property ${ }^{13}$. However, it satisfies similar properties. Instead of irrelevant player independence, the conjunctive permission value satisfies inessential player independence, requiring that payoffs of essential players do not depend on the presence of inessential players (instead of requiring that payoffs of relevant players do not depend on the presence of irrelevant players). Let $\operatorname{Iness}(N, v, D)$ be the set of inessential players in $(N, v, D)$.

\footnotetext{
${ }^{10}$ Similarly, the irrelevant player property is superfluous in Theorem 3.4.

${ }^{11}$ For games with a permission structure, these axioms are defined the same as for games under precedence constraints, by simply replacing the domain $\mathcal{G}_{P C}$ by the domain $\mathcal{G}_{P S}$ in the definitions in the previous sections.

${ }^{12}$ This is a weaker version of necessary player symmetry used by van den Brink et al. (2015) for the more specific permission tree games, requiring that all necessary players in the game earn the same payoff irrespective of their position in the digraph.

${ }^{13}$ Also stating these axioms for games with a permission structure, we can simply replace the domain $\mathcal{G}_{P C}$ by the domain $\mathcal{G}_{P S}$, but we also need to redefine what is a null player as a player whose marginal contribution is zero to any coalition in $2^{N}$.
} 
Inessential player independence For every $(N, v, D) \in \mathcal{G}_{P S}$, it holds that $f_{i}(N, v, D)=$ $f_{i}\left(N^{\prime}, v_{N^{\prime}}, D\left(N^{\prime}\right)\right)$ for $i \in N^{\prime}$, with $N^{\prime}=N \backslash \operatorname{Iness}(N, v, D)$.

In a similar way, the irrelevant player property can be modified. This gives the inessential player property that requires that a null player whose subordinates are all null players, earns a zero payoff. Similar as in Proposition 4.3, efficiency and inessential player independence imply the inessential player property.

Note that, similar as the irrelevant player property, the inessential player property is weaker than the null player property. The null player property deals with all players who are null players in the game, while the inessential player property only takes care of the null players whose subordinates are also null players. For permission tree games, van den Brink et al. (2015) deal with this by the axiom which requires that the payoff distribution does not change if a predecessor $i$ becomes necessary for its successor $j$ in the sense that the marginal contribution of player $j$ to every coalition that does not contain player $i$ becomes zero. For game $(N, v)$ and players $i, j \in N$, the game $\left(N, v_{j}^{i}\right)$ is defined by $v_{j}^{i}(S)=v(S \backslash\{j\})$ for all $S \subseteq N \backslash\{i\}$, and $v_{j}^{i}(S)=v(S)$ otherwise.

Predecessor necessity For every $(N, v, D) \in \mathcal{G}_{P S}$ and $i, j \in N$ such that $(i, j) \in D$, it holds that $f(N, v, D)=f\left(N, v_{j}^{i}, D\right)$.

Although predecessor necessity is used for axiomatization on permission tree games, it is also satisfied for all games with an acyclic permission structure.

Next, we give a new characterization of the conjunctive permission value that uses similar axioms as used to characterize the precedence Shapley value and the hierarchical solution for games under precedence constraints.

Theorem 4.5 A solution for games with acyclic permission structure is equal to the conjunctive permission value $\varphi^{c}$ if and only if it satisfies efficiency, linearity, inessential player independence, predecessor necessity and equal-strength.

Proof:

It is straightforward to verify that the conjunctive permission value satisfies the five axioms. To prove uniqueness, suppose that solution $f$ for games with an acyclic permission structure satisfies the five axioms. Consider any $(N, D) \in \mathcal{D}$ and $\emptyset \neq T \subseteq N$.

Efficiency and inessential player independence imply the inessential player property, and thus

$$
f_{i}\left(N, u_{T}, D\right)=0 \text { for all } i \in N \backslash\left(T \cup \widehat{P}_{D}(T)\right) .
$$

Repeated application of predecessor necessity implies that $f\left(N, u_{T}, D\right)=f\left(N, u_{T \cup \widehat{P}_{D}(T)}, D\right)$. 
Inessential player independence implies that for all $i \in T \cup \widehat{P}_{D}(T)$

$$
f_{i}\left(N, u_{T \cup \widehat{P}_{D}(T)}, D\right)=f_{i}\left(T \cup \widehat{P}_{D}(T), u_{T \cup \widehat{P}_{D}(T)}, D\left(T \cup \widehat{P}_{D}(T)\right)\right) .
$$

Equal strength implies that there is an $\alpha \in \mathbb{R}$ such that

$$
f_{i}\left(T \cup \widehat{P}_{D}(T), u_{T \cup \widehat{P}_{D}(T)}, D\left(T \cup \widehat{P}_{D}(T)\right)\right)=\alpha \text { for all } i \in T \cup \widehat{P}_{D}(T) .
$$

Efficiency then implies that $\alpha=\frac{1}{\left|T \cup \widehat{P}_{D}(T)\right|}$.

With (4.12) it then follows for all $i \in T \cup \widehat{P}_{D}(T)$,

$$
f_{i}\left(N, u_{T \cup \widehat{P}_{D}(T)}, D\right)=f_{i}\left(T \cup \widehat{P}_{D}(T), u_{T \cup \widehat{P}_{D}(T)}, D\left(T \cup \widehat{P}_{D}(T)\right)\right)=\frac{1}{\left|T \cup \widehat{P}_{D}(T)\right|}
$$

With (4.11) then $f\left(N, u_{T}, D\right)$ is determined.

Since efficiency and inessential player independence imply the inessential player property, for a null game $v^{0}(S)=0$ for all $S \subseteq N$, and thus we have that $f_{i}\left(N, v^{0}, D\right)=0$ for all $i \in N$ and $(N, D) \in \mathcal{D}$.

Since $f$ satisfies linearity, the solution $f(N, v, D)$ is uniquely determined and coincides with the conjunctive permission value, for any $(N, v, D) \in \mathcal{G}_{P S}$.

Now, an obvious next question is if we can generalize the equal strength to $p$-strength also in this context. This can be done, and it yields the following class of solutions where the Harsanyi dividends in the conjunctive restricted game are allocated proportional to a network power measure.

Definition 4.6 For positive power measure $p$, the p-permission value is the solution for games with a permission structure given by

$$
\begin{aligned}
\widehat{H}_{i}^{p}(N, v, D) & =\sum_{\substack{S \subseteq N \\
i \in S}} \frac{p_{i}(S, D(S))}{\sum_{j \in S} p_{j}(S, D(S))} \Delta_{r_{v, D}^{c}}(S) \\
& =\sum_{\substack{S \in \Phi^{c}(N, D) \\
i \in S}} \frac{p_{i}(S, D(S))}{\sum_{j \in S} p_{j}(S, D(S))} \Delta_{r_{v, D}^{c}}(S) \text { for all } i \in N .
\end{aligned}
$$

A main difference with the precedence power solutions is that now we consider the Harsanyi dividends in the conjunctive restricted game $r_{v, D}^{c}$ instead of the original game $v$ on the domain. 
Theorem 4.7 A solution for games with an acyclic permission structure is equal to the p-permission value $\widehat{H}^{p}$ if and only if it satisfies efficiency, linearity, inessential player independence, predecessor necessity and p-strength.

The proof of uniqueness follows straightforward from the proof of Theorem 4.5 by replacing (4.13), which followed from equal strength, applying $p$-strength:

$$
p_{i}(N, D) f_{j}\left(N,, u_{N}, D\right)=p_{j}(N, D) f_{i}\left(N, u_{N}, D\right)
$$

when $(N, D)=\left(T \cup \widehat{P}_{D}(T), D\left(T \cup \widehat{P}_{D}(T)\right)\right)$. Together with efficiency, this determines the payoffs in $f\left(T \cup \widehat{P}_{D}(T), u_{T \cup \widehat{P}_{D}(T)}, D\left(T \cup \widehat{P}_{D}(T)\right)\right)$. We will refer to the solutions characterized in Theorem 4.7 as permission power solutions. Logical independence of the axioms is again shown in Section 5.

An interesting question is now also to see which precedence power solution on $\mathcal{G}_{P C}$ satisfies equal strength, i.e. the $p$-strength axiom with the equal power measure that is used to characterize the conjunctive permission value on $\mathcal{G}_{P S}$ in Theorem 4.5. It turns out that this gives essentially the Shapley value, i.e. the solution $\widetilde{S h}$ on $\mathcal{G}_{P C}$ that to every game under precedence constraints assigns the Shapley value of the unrestricted game (extended to the power set of $N$ ), i.e.

$$
\widetilde{S h}_{i}(N, v, D)=\operatorname{Sh}(N, \bar{v}) \text { for all }(N, v, D) \in \mathcal{G}_{P C}
$$

where $\bar{v} \in \mathcal{G}^{N}$ is given by $\Delta_{\bar{v}}(S)=\Delta_{v}^{D}(S)$ if $S \in \Phi^{p}(N, D)$, and $\Delta_{\bar{v}}(S)=0$ if $S \notin \Phi^{p}(N, D)$.

\section{Logical Independence}

In this section, we present alternative solutions for games with a hierarchy, that show logical independence of the axioms in the main theorems in Section 4.

\subsection{Logical Independence of the Axioms in Theorem 4.4}

The following alternative solutions each satisfy all but one of the axioms in Theorem 4.4.

1. Consider the solution $f^{z e r o}$ on $\mathcal{G}_{P C}$ that always assigns zero payoff to every player in every game under precedence constraint, i.e.

$$
f_{i}^{z e r o}(N, v, D)=0 \text { for all } i \in N \text { and }(N, v, D) \in \mathcal{G}_{P C} .
$$

This solution satisfies all axioms of Theorem 4.4 except efficiency. 
2. For positive power measure $p$, consider the solution $f$ on $\mathcal{G}_{P C}$ that allocates the dividend of every coalition proportional to the power measure $p$ among all relevant players, i.e., for all $i \in N$ and $(N, v, D) \in \mathcal{G}_{P C}$

$$
f_{i}(N, v, D)=H_{i}^{p}\left(N, v(N) u_{\bigcup_{\left\{T \in \Phi^{p}(N, D), \Delta v_{(T) \neq 0\}}\right.} T}, D\right) .
$$

This solution satisfies all axioms of Theorem 4.4 except linearity.

3. For positive power measure $p$, consider the solution $f$ on $\mathcal{G}_{P C}$ that allocates the dividends of every coalition in every game under precedence constraints proportional to the power values $p(N, D)$, i.e. for positive power measure $p$,

$$
f_{i}^{p}(N, v, D)=\sum_{\substack{S \in \Phi^{p}(N, D) \\ i \in S}} \frac{p_{i}(N, D)}{\sum_{j \in S} p_{j}(N, D)} \Delta_{v}^{D}(S) \text { for all } i \in N .
$$

Compared to the precedence power solutions, these solutions allocate the dividend of coalition $S$ proportional to the power values $p(N, D)$ in the original digraph instead of the power values $p(S, D(S))$ in the subgraphs on $S$. This solution satisfies all axioms of Theorem 4.4 except irrelevant player independence.

4. Let the equal network power measure $\gamma$ be given by $\gamma_{i}(N, D)=\frac{1}{|N|}$ for all $i \in N$ and $(N, D) \in \mathcal{D}$. For $p \neq \gamma$, consider the solution $\widetilde{S h}=H^{\gamma}$ on $\mathcal{G}_{P C}$. This solution satisfies all axioms of Theorem 4.4 except $p$-strength. For $p=\gamma$, the hierarchical solution satisfies all axioms of Theorem 4.4 except $\gamma$-strength.

\subsection{Logical Independence of the Axioms in Theorem 4.7}

The following alternative solutions each satisfy all but one of the axioms in Theorem 4.7.

1. Solution $f^{z e r o}$ assigning zero payoff to every player in every game with a permission structure, satisfies all axioms of Theorem 4.7 except efficiency.

2. For positive power measure $p$, consider the solution $f$ for games with a permission structure that allocates the dividend of every coalition in the conjunctive restricted game proportional to the power measure $p$ among all essential players, i.e. for all $i \in N$ and $(N, v, D) \in \mathcal{G}_{P S}$,

$$
f_{i}(N, v, D)=\widehat{H}_{i}^{p}\left(N, v(N) u_{\left.\bigcup_{\left\{T \subseteq N, \Delta_{r}^{c}\right.}(T, D) \neq 0\right\}} T, D\right) .
$$

This solution satisfies all axioms of Theorem 4.7 except linearity. 
3. For positive power measure $p$, consider the solution $f$ for games with a permission structure that allocates the dividends of every coalition in the conjunctive restricted game proportional to the power values $p(N, D)$, i.e. for positive power measure $p$,

$$
f_{i}^{p}(N, v, D)=\sum_{\substack{S \subseteq N \\ i \in \mathcal{S}}} \frac{p_{i}(N, D)}{\sum_{j \in S} p_{j}(N, D)} \Delta_{r_{v, D}^{c}}(S) \text { for all } i \in N .
$$

This solution satisfies all axioms of Theorem 4.7 except inessential player independence.

4. For positive power measure $p$, consider the solution that allocates the dividend of every coalition $S$ in the original game proportional to the power measure $p(S, D(S))$ in the subgraph on $S$, i.e.

$$
\widetilde{H}_{i}^{p}(N, v, D)=\sum_{\substack{S \subseteq N \\ i \in S}} \frac{p_{i}(S, D(S))}{\sum_{j \in S} p_{j}(S, D(S))} \Delta_{v}(S) \text { for all } i \in N
$$

This solution satisfies all axioms of Theorem 4.7 except predecessor necessity.

5. For $p \neq \gamma$, the conjunctive permission value satisfies all axioms of Theorem 4.7 except $p$-strength. For $p=\gamma$, the $p$-permission power value with $p=h$ being the hierarchical strength, satisfies all axioms of Theorem 4.7 except $\gamma$-strength.

\section{Conclusions}

The goal of this chapter is to review and compare two well-known approaches to games with a hierarchy in the literature: the permission structure approach and the precedence constraint approach. Moreover, by a new axiomatization of the conjunctive permission value, we could extend this solution and its axiomatization to define the new class of permission power solutions which is characterized by axioms that make it comparable with the class of precedence power solutions.

There are several extensions of the model that can be considered. For example, instead of digraphs other combinatorial structures might represent some relational structure among the players. A 'natural' extension of games with a permission structures are games on antimatroids see Algaba et al. (2003, 2004b). Antimatroids are combinatorial structures introduced by Dilworth (1940), see also Edelman and Jamison (1985) which, besides permission structures, also generalize other models such as ordered partition voting where players are partitioned into levels, and a coalition in a certain level can be active only if a majority of players in higher levels approve. Since antimatroids are union closed 
(i.e. the union of any two feasible coalitions is also feasible), a similar approach for games with a permission structure can be followed by defining a restricted game that assigns to every coalition the worth of its largest feasible subset in the original game, and applying the Shapley value (or any other TU-game solution) to this restricted game. Different extensions of the Shapley value for games on union closed systems are considered in van den Brink et al. (2011b). An even more general model are games on union stable systems, see Algaba et al. (2000, 2001a), and Algaba et al. (2001b, 2004a), where feasibility of the union of two feasible coalitions is only required if the two coalitions have a non-empty intersection, which reflects the communication feature. In this framework, Algaba et al. (2015) applied power measures to distribute dividends in games on union stable systems extending some results given in Algaba et al. (2012) about the Myerson and position values. Network structures taking into account both hierarchical and communication features have been introduced in Algaba et al. (2018). A 'natural' structure to extend games under precedence constraints are augmenting systems (see Bilbao (2003) and Algaba et al. (2010)) and regular set systems (see Honda and Grabisch (2006) and Lange and Grabisch (2009)).

In this chapter, (i) we recall one axiomatization of the conjunctive permission value, and one from the precedence Shapley value from the literature, (ii) we applied network power measures to define two classes of solutions from them, and (iii) developed these axiomatizations further into two comparable axiomatizations of these two classes. Further work can be done to see if other types of axioms can be part of comparable axiomatizations, such as axiomatizations using some type of fairness axiom (see van den Brink (1997) for games with a permission structure, Algaba et al. (2003) for games on antimatroids, and Algaba et al. (2001a) for games on union stable systems). Further research will include the introduction and analysis of the disjunctive permission approach in the context of games under precedence constraints.

The connection between game theoretic payoff allocation and social network power measures gives insight in different solutions which might be helpful in applications. In this chapter, on one hand, within each of these two classes of solutions, different network power measures yield different solutions from the class. But on the other hand, taking one specific power measure we obtain two solutions, one from each class.

In this chapter, we applied power measures to define solutions for cooperative games with a hierarchical network structure. The other way around, solutions for games on (directed or undirected) networks can be used to measure power or centrality in networks. Taking a (symmetric) game and applying a solution for graph games yields such a power measure. For example, Gómez et al. (2003) apply the Myerson value for communication graph games to certain symmetric games restricted on an undirected graph in the sense of 
Myerson (1977), measuring different types of centrality in undirected graphs. Similar, we can apply the precedence power or permission power solutions to games with a hierarchical structure to obtain power measures for acyclic digraphs. Obviously, when we use the unanimity game of the grand coalition, we get the same power measure that we use for the solution. But, as done in Gómez et al. (2003) for undirected graphs, other games can be used.

Without going into power measurement, notice that network centrality has a very different effect in the precedence approach than in the permission approach. Consider a strict hierarchical network that is represented by a linear order $\left\{\left(i_{k}, i_{k+1}\right) \mid k=1, \ldots n-1\right\}$. Both in the permission as well as the precedence approach, the top player $i_{1}$ is 'powerful' in obtaining a share in the worth of the game. However, there is a difference considering the networks $\Phi^{c}(N, D)$ and $\Phi^{p}(N, D)$. Since $\Phi^{c}(N, D)=\left\{\left\{i_{1}, \ldots, i_{l}\right\} \mid l=1, \ldots, n\right\}$, the top player $i_{1}$ can be considered to be the most central since it belongs to every feasible coalition. However, since $\Phi^{p}(N, D)=\left\{\left\{i_{l}, \ldots, i_{n}\right\} \mid l=1, \ldots, n\right\}$, in the precedence approach the top player $i_{1}$ seems to be the least central since the only feasible coalition it belongs to is the grand coalition. On the other hand, the bottom player $i_{n}$ seems to be the most central and belongs to every feasible coalition. Notice that in the precedence approach, being a bottom player means that you are feasible as a singleton, but then you can be the first player to enter in an admissible permutation, and if you have a predecessor in the hierarchy, you will never be the last to enter which is disadvantageous in, for example, convex games. On the other hand, being in few feasible coalitions means that you more often enter as the last player, which in many games (in particular in convex games) gives you a benefit.

\section{Acknowledgments}

Financial support from the Government of Spain (Ministerio de Economía y CompetitividadMINECO) and FEDER under the project MTM2014-54199-P is gratefully acknowledged.

\section{References}

[1] Algaba, E., Bilbao, M., Borm, P., López, J. (2000) The position value for union stable systems. Mathematical Methods of Operations Research 52, 221-236.

[2] Algaba, E., Bilbao, M., Borm, P., López, J. (2001a) The Myerson value for union stable structures. Mathematical Methods of Operations Research 54, 359-371. 
[3] Algaba, E., Bilbao, M. López, J. (2001b) A unified approach to restricted games. Theory and Decision 50, 333-345.

[4] Algaba, E., Bilbao, M., van den Brink, R, Jiménez-Losada, A. (2003) Axiomatizations of the Shapley value for games on antimatroids. Mathematical Methods of Operations Research 57, 49-65.

[5] Algaba, E., Bilbao, J.M., López, J. (2004a) The position value in communication structures. Mathematical Methods of Operations Research 59, 465-477.

[6] Algaba, E., Bilbao, M., van den Brink, R, Jiménez-Losada, A. (2004b) Cooperative games on antimatroids. Discrete Mathematics 282, 1-15.

[7] Algaba, E., Bilbao, J. M, Slikker, M. (2010) A value for games restricted by augmenting systems. SIAM Journal on Discrete Mathematics 24, 992-1010.

[8] Algaba, E., Bilbao, J.M, van den Brink, R., Lopez J.J. (2012) The Myerson value and superfluous supports in union stable systems. Journal of Optimization Theory and Applications 155, 650-668.

[9] Algaba, E., Bilbao,, J. van den Brink, R. (2015) Harsanyi power solutions for games on union stable systems. Annals of Operations Research 225, 27-44.

[10] Algaba, E., van den Brink, R., Dietz, C. (2017) Power measures and solutions for games under precedence constraints. Journal of Optimization Theory and Applications 172, 1008-1022.

[11] Algaba, E., van den Brink, R., Dietz, C. (2018) Network structures with hierarchy and communication. Journal of Optimization Theory and Applications 179(1), 265-282.

[12] Bilbao, J.M., Edelman, P.H. (2000) The Shapley value on convex geometries. Discrete Applied Mathematics 103, 33-40.

[13] Bilbao, J. M. (2003) Cooperative games under augmenting systems. SIAM Journal on Discrete Mathematics 17, 122-133.

[14] Borm, P., Owen, G., Tijs, S. (1992) On the position value for communication situations, SIAM Journal on Discrete Mathematics 5, 305-320.

[15] Brink R. van den, Gilles, R.P. (1996) Axiomatizations of the Conjunctive Permission Value for Games with Permission Structures. Games and Economic Behavior 12, 113-126.

[16] Brink, R. van den, (1997) An Axiomatization of the Disjunctive Permission Value for Games with a Permission Structure International Journal of Game Theory 26, 27-43. 
[17] Brink R. van den, Laan G. van der, Pruzhansky, V. (2011a) Harsanyi power solutions for graph-restricted games. International Journal of Game Theory 40, 87-110.

[18] Brink, R. van den, Katsev, I, van der Laan, G. (2011b) Axiomatizations of Two Types of Shapley Values for Games on Union Closed Systems. Economic Theory 47, 175-188.

[19] Brink, R. van den, P.J.J. Herings, G. van der Laan, and A.J.J. Talman (2015) The Average Tree Permission Value for Games with a Permission Tree. Economic Theory 58, $99-123$.

[20] Dilworth, R. P. (1940) Lattices with Unique Irreducible Decompositions. Annals of Mathematics 41, 771-777.

[21] Edelman, P. H., Jamison, R. E. (1985) The Theory of Convex Geometries. Geometrica Dedicata 19, 247-270.

[22] Faigle, U., Kern, W. (1992) The Shapley Value for Cooperative Games under Precedence Constraints. International Journal of Game Theory 21, 249-266.

[23] Gilles, R.P., Owen, G., and van den Brink, R. (1992) Games with permission structures: the conjunctive approach. International Journal of Game Theory 20, 277-293.

[24] Gilles, R. P., Owen, G. (1994) Cooperative games and disjunctive permission structures, Department of Economics, Virginia Polytechnic Institute and State University, Blacksburg, Virginia.

[25] Gilles, R. P. (2010) The Cooperative Game Theory of Networks and Hierarchies, Springer Verlag, Berlin Heidelberg.

[26] Gómez D., González-Arangüena, E., Manuel, C., Owen, G. del Pozo, M., Tejada, J. (2003) Centrality and power in social networks: a game theoretic approach. Mathematical Social Sciences 46, 27-54.

[27] Harsanyi, J.C. (1959) A bargaining model for cooperative n-person games. In: Tucker, A.W., Luce, R.D. (eds.): Contributions to the Theory of Games IV, pp. 325-355. Princeton University Press, Princeton, NJ.

[28] Honda, A., Grabisch, M. (2006) Entropy of capacities on lattices and set systems. Information Sciences 176, 3472-3489.

[29] Lange, F., Grabisch, M. (2009) Values on regular games under Kirchhoff's laws. Mathematical Social Sciences 58, 322-340 
[30] Myerson, R. B. (1977) Graphs and cooperation in games. Mathematics of Operations Research 2, 225-229.

[31] Shapley, L.S. (1953) A Value for $n$-Person Games. In: Kuhn, H.W., Tucker, A.W. (eds.): Contributions to the Theory of Games Vol.2, pp.307-317, Princeton UP, Princeton. 